somewhat with the discovery ${ }^{6}$ that gas concentrations in bottom waters are rising again after the release, and there is now general agreement that the dissolved gas should be artificially removed from the lake by the installation of pipes $^{7}$. However, the long-missing mathematical model of an eruption fountain is a valuable new tool in assessing the hazards associated with gassy lakes, and any possible remedies.

Zhang argues that for a fountain to persist for any length of time and yet remain confined, the pressure profile inside the rising conduit must be near the hydrostatic gradient in the surrounding water column. The required flow conditions are thus different from those of fluid movement in pipes, where the walls allow large pressure imbalances. A fountain exit velocity can be calculated for any assumed $\mathrm{CO}_{2}$ concentration (or corresponding saturation depth), and so Zhang's treatment can be applied directly to the general case of a gas-charged lake where instability could develop at any depth below the chemocline ${ }^{8}$, the density boundary that prevents deeper seasonal flushing.

Regarding the 1986 Lake Nyos disaster, for a proposed maximum $\mathrm{CO}_{2}$ concentration in bottom water of $0.43 \mathrm{~mol}$ $\mathrm{kg}^{-1}$ (ref. 9), fountain heights in excess of $150 \mathrm{~m}$ could be reached. Decompressed to surface pressure, such fluid would theoretically have a porosity of 90 per cent, which is above the point of transition from bubbly flow to a stream of fragmented water droplets ${ }^{2,10}$. During its most violent phase the fountain would thus resemble a scaled-up geyser more than an uncorked champagne bottle, the resultant mist in the moonlight possibly looking very much as one eye-witness described", like "smoke come out of lake".

William C. Evans is at the US Geological Survey, Menlo Park, California 94025, USA.

\title{
Avalanche theory in rice
}

\section{Mehran Kardar}

BY patiently dropping grains of rice, in the experiment reported on page 49 of this issue, Frette et al. ${ }^{1}$ put to the test some features of 'self-organized criticality' (SOC), a purported generic mechanism for the common occurrence of fractals, $1 / f$-noise and power-law distributed events. Although SOC is frequently observed in numerical simulations, controlled experiments on granular media have at best provided ambiguous results. Some piles of sand relax by big avalanches (of the order of the system size), whereas the statistics of avalanches in others can be fitted to different distributions. Depending on the shape of their rice grains, Frette et al. obtain both types of behaviour: small aspect ratios (short, fat grains) lead to large avalanches, larger ones (longer grains) lead to a power-law distribution for avalanche sizes - the signature of self-organized criticality.

A common exercise in elementary physics is to estimate the typical value of some quantity by dimensional analysis. Yet there exist many phenomena that are effectively scale-free, with possible values that span several orders of magnitude. Fractals - geometrical entities whose fluctuations repeat in self-similar fashion from the smallest to largest distances ${ }^{2}-$ have been invoked to characterize natural shapes from coastlines to clouds. Possibly related manifestations of selfsimilarity in the time domain are the broad-band noise observed in electrical circuits, and the fluctuations in time of the flow of rivers, or light from quasars. One model of this so-called $1 / f$-noise relies on the incoherent superposition of signals from oscillators with a wide (scale-free) distribution of frequencies $f$. Power-law distributions also seem to characterize the size (strength) of many natural catastrophes: avalanches, mass extinctions and stock-market crashes, to name but a few. The Gutenberg-Richter law, which relates the number of earthquakes to their magnitude, is a prominent example ${ }^{3}$.

Why are scale-free phenomena so frequent in nature? In 1987, Bak, Tang and Wiesenfeld ${ }^{4}$ introduced the idea of selforganized criticality as a possible answer. They used the analogy of a pile of sand gradually built up by the addition of single grains. Once the pile has reached a critical slope, addition of further grains may cause avalanches, while the overall pile fluctuates around this 'angle of repose'. In the idealized sand pile, modelled on the computer, avalanches of all sizes occur at this stage, with a probability distribution that is scalefree (a power law).

The initial claims of SOC as the underlying link between fractals, $1 / f$ noise and power-law distributed events naturally excited the imagination of a broad spectrum of scientists. Currently, the principle is applied more modestly to explain power-law distributions of events (avalanches) observed in some slowly driven systems. Earthquakes, forest fires and rainfalls are just some of the diverse phenomena that have been suggested to show SOC.

As the original theory was cast in terms of avalanches on a sand pile, granular systems have been a prime target in the experimental search for SOC. But the behaviour of real sand is complex, showing a variety of slip, slide and roll motion not usually incorporated in the simple dissipative computer models. Some piles relax in large avalanches where a top layer of sand slides away ${ }^{5}$, and even though a spectrum of avalanche sizes is observed in other experiments ${ }^{6,7}$, the limited range of data makes a convincing identification of SOC difficult.

Frette et al. set out to do the definitive experiment on this subject, and they have carried out the task with great patience and care. They do their experiment on grains of rice, which they find to be less subject to slipping and rolling than are sand grains, and hence closer to the dissipative limit studied numerically. (This is especially true for long-grain rice, with its larger aspect ratio.) They accumulate sufficient statistics to distinguish between a power-law distribution in one case (long grains) and a stretched exponential in another (more spherical grains). The occurrence of both behaviours in the same experiment is valuable, and allows the authors to provide possible reasons for distinct relaxation dynamics by appealing to the relative importance of inertia and friction. Although this explanation is not new, here it is clearly demonstrated.

Whether or not SOC is indeed a useful model (and the debate is not yet settled), it has certainly generated broad excitement. The new experiment provides the best evidence so far for the occurrence (sometimes, but not always) of powerlaw distributed avalanches in granular media. The continuing challenge to both theorists and experimentalists is to go beyond observation of power-law distributed events to the identification of the exponent and demonstration of its universality in different systems - that is, to go from qualitative to quantitative agreement.

Mehran Kardar is in the Department of Physics, Massachusetts Institute of Technology, Cambridge, Massachusetts 02139, USA.

1. Frette, V. et al. Nature 379, 49-52 (1996).

2. Mandelbrot, B. B. The Fractal Geometry of Nature (Freeman, San Francisco, 1982).

3. Gutenberg, B. \& Richter, C. F. Ann. Geofis. 9, 1 (1956)

4. Bak, P., Tang, C. \& Wiesenfeld, K. Phys. Rev. Lett. 59 , 381-384 (1987)

5. Jaeger, H. M., Liu, C.-H. \& Nagel, S. R. Phys. Rev. Lett. 62, 40-43 (1989).

6. Held, G. A. et al. Phys. Rev. Lett. 65, 1120-1123 (1990).

7. Bretz, M., Cunningham, J. B., Kurczynski, P. L. \& Nori, F. Phys. Rev. Lett. 69, 2431-2434 (1992). 